\title{
Handling KDD Process Changes by Incremental Replanning
}

\author{
Ning Zhong ${ }^{1}$, Chunnian Liu ${ }^{2}$, Yoshitsugu Kakemoto ${ }^{3}$, and Setsuo Ohsuga ${ }^{4}$ \\ 1 Dept. of Computer Science and Sys. Eng., Yamaguchi University \\ 2 Dept. of Computer Science, Beijing Polytechnic University \\ 3 RCAST, The University of Tokyo \\ 4 Dept. of Information and Computer Science, Waseda University
}

\begin{abstract}
Within the framework of our GLS (Global Learning Scheme) system that is a multi-strategy and cooperative KDD (Knowledge Discovery in Databases) system, this paper reports new research progress, by addressing one deeper issue concerning KDD process planning: change management, and giving our solution for it. The problem on change management can be largely solved by an incremental replanning technique. With the issue being properly handled, the GLS system is more complete in KDD process modeling, and more flexible and robust for practical use.
\end{abstract}

\section{Introduction}

Our previous work in the GLS system has set up the KDD process framework as an organized society of intelligent agents, and solved the basic problem in a multi-strategy and cooperative KDD system: how to choose appropriate KDD techniques to achieve a particular discovery goal in a particular domain $[9,10]$. The solution has been based on AI planning techniques, and increased both autonomy and versatility of the GLS system [11, 12]. However, KDD process and its planning is a new research field, there are more challenging and deeper issues demanding further research such as

- How to manage changes in a KDD process?

- How to handle KDD process iteration?

In particular, during the (long) lifetime of a KDD application session, there may be many kinds of changes which demand replanning the KDD process, such as changes in the databases, introducing new KDD techniques and/or new strategies to coordinate various discovery steps, etc. As replanning from scratch is in most cases unpleasant and unnecessary, we need a method to reuse the exiting KDD process plan, with local adjustment adapted to the changes.

The major contributions of this paper are addressing and solving the first one of the two important issues that have been essentially untouched in KDD literature, that is, handling KDD process changes by incremental replanning. The second problem (process iteration) can be solved by a mechanism integrating process planning with process controlling. We would like to discuss this problem in our other papers. The remaining part of this paper is organized as follows: 
Section 2 gives a short summary of the GLS system and its planner; Sections 3 describes how to handle KDD process change by incremental replanning techniques; Finally Section 4 gives the concluding remarks.

\section{An Overview of GLS and Its Planner}

In this section, we give a brief summary of the GLS architecture and its planner. Here we only present the materials which are strictly needed in understanding the core part of the paper. The reader may refer to (Zhong et al 1997a; Zhong et al $1997 \mathrm{~b}$, Zhong et al 1997c) for more details.

\subsection{GLS Architecture}

The GLS system is divided into three levels: two meta-levels and one object level. On the first meta-level, the planning meta-agent (KDD planner, for short) sets the discovery process plan that will achieve the discovery goals when executed. On the second meta-level, the KDD agents are dynamically generated, executed, and controlled by the controlling meta-agent (KDD controller, for short). On the object level, the KDD agents are grouped into three learning phases: (1) Preprocessing, (2) Knowledge-elicitation, (3) Knowledge-refinement. Each phase includes a set of KDD techniques modeled as KDD agents, such as $C B K, Q D R$, $F S N, S C T$ in phase (1); KOSI, DBI, GDT-RS in phase (2); and IIBR, HML in phase (3). Each KDD agent represents a simple discovery step, and when they are organized into a society, more complex discovery tasks can be accomplished. The KDD planner is just a meta-tool to automatically build such a society.

\subsection{Formal Description of KDD Agents}

In terms of AI planning, a KDD agent is an operator. We must formally describe the KDD agents as operators in order to apply AI planning techniques here. The formalism for this purpose is the OOER (Object-Oriented Entity Relationship) data model, which extends the traditional ER concepts (entity/relation, type/instance, instance-level attributes) with object-oriented concepts (subtyping, multiple inheritance, procedures, type-level attributes/procedures, and so on).

There are two kinds of types: $D \mathscr{G} K$ types (describing passive objects - various data and knowledge presented in a KDD system) and Agent types (describing active objects - various KDD techniques used in the GLS system). Types are organized into a hierarchy by the OO subtyping mechanism. DEKK and Agent are the supertypes for all passive and active objects respectively. In the text of this paper, an "Agent type" means any subtype of Agent, modeling a particular KDD technique.

Types have ordinary instance-level attributes. As for Agent types, there are additional properties defined as "type-level" attributes with information that is used by the planning meta-agent: 
- In/Out: specifying the types of the input/output of an Agent type.

- Precond/Effect: specifying the preconditions for an agent (an instance of the Agent type) to execute, and the effects when executed. Note that a large part of the Precond/Effect, concerning constraints on input/output of the Agent type, has been specified implicitly by the In/Out attribute.

- Action: a sequential program performing real KDD actions upon agent execution.

- Decomp: describing possible subtasking. When a "high-level" agent (with dummy Action) is to be executed, it is decomposed into a network of subagents (i.e. a subplan). Decomp specifies the candidate Agent types for the subagents.

\subsection{The KDD Planner}

AI planning in general works on the goal, the pool of operators, and the world state descriptions (WSD). In KDD planning, the goal is a knowledge discovery task, the operators are the KDD Agent types, and the WSD is the description of the structure and status of the databases (the source of learning) and the knowledge bases (the results of learning). Given a discovery goal, the KDD planner reasons on operators and WSD to build a KDD process plan - a network of KDD agents that will achieve the discovery goal when executed. It has three layers as shown in Figure 1.

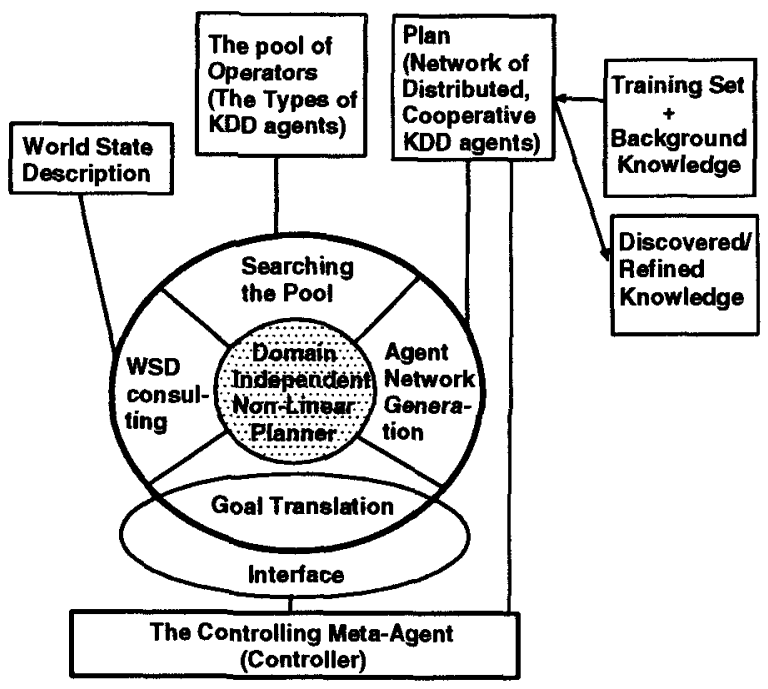

Fig. 1. The planning meta-agent coupled with the controlling meta-agent 
- The Inner Layer: A Domain-Independent Non-Linear Planner.

Given the initial world state description (WSD), the discovery goal and the pool of operators (KDD agents), it starts with a dummy partial plan, then expands the partial plan until finds a complete and consistent plan that achieves the goal when executed. The Planner is implemented by a production system which will be extended/modified in this paper to cope with new issues (iteration/changes). We will (re)present its original (meta-)rule set and searching strategy before introducing any extension/modification in the following sections.

- The Outer Layer: The Interface with the KDD Controller to Realize Hierarchical Planning.

Hierarchical planning is necessary for real-world application, which means alternatively adding detailed steps to the plan and actually executing some steps until the overall goal is achieved. In GLS, this is accomplished by the cooperation of the two meta-agents - planner and controller. The interface between them is the outer layer.

- The Middle Layer: KDD Specific Issues.

Because the core of the planner is domain-independent, we provide a middle layer to deal with all KDD specific issues including: (1) To transform the KDD goals into AI goals (conjunctions of logical literals); (2) To search the pool of operators for suitable KDD agents achieving a subgoal, hence being introduced into the plan; (3) To consult the world state description (WSD) to see if a subgoal is already satisfied by the WSD, or to translate the In/Out specification in an agent into logical literals that are added to Precond/Effect of the agent; (4) To represent the resulting plan as a network of KDD agents, so the controller can dynamically generate and execute the KDD agents according to the network.

\subsection{A Scenario}

Assume that we have a central, large space science database, each record (tuple) describing a star. The interesting attributes include CD (cluster designation), ET (effective temperature), LU (luminosity), B-V and U-B (color indexes). The facts such as we have already had a central, large database with CleanData, and the nominal attribute CD can be used for forming Scopes, etc. are explicitly stated in the initial-state (WSD). The discovery goal is to find structural characteristics hidden in the database and to refine them upon data change. Based on the specifications of WSD, goal, and KDD agent types, the planner and the controller cooperate and come up with a full KDD process plan as shown in Figure 2.

The process goes as follows. The initial plan consists of a single KDD agent Kdiscover to produce RegreTree that is a subtype of Krefined. It is decomposed into the sequential phases: Preprocess, Kelicit, and Krefine (in Figure 2, we also show the input/output types for these KDD agents). As the WSD contains the fact that we have already got CleanData, Preprocess can be simply done by Select (no need of Collect and Clean). Because the nominal attribute CD designates star clusters, and we need clustering other attributes as preparation for the next 


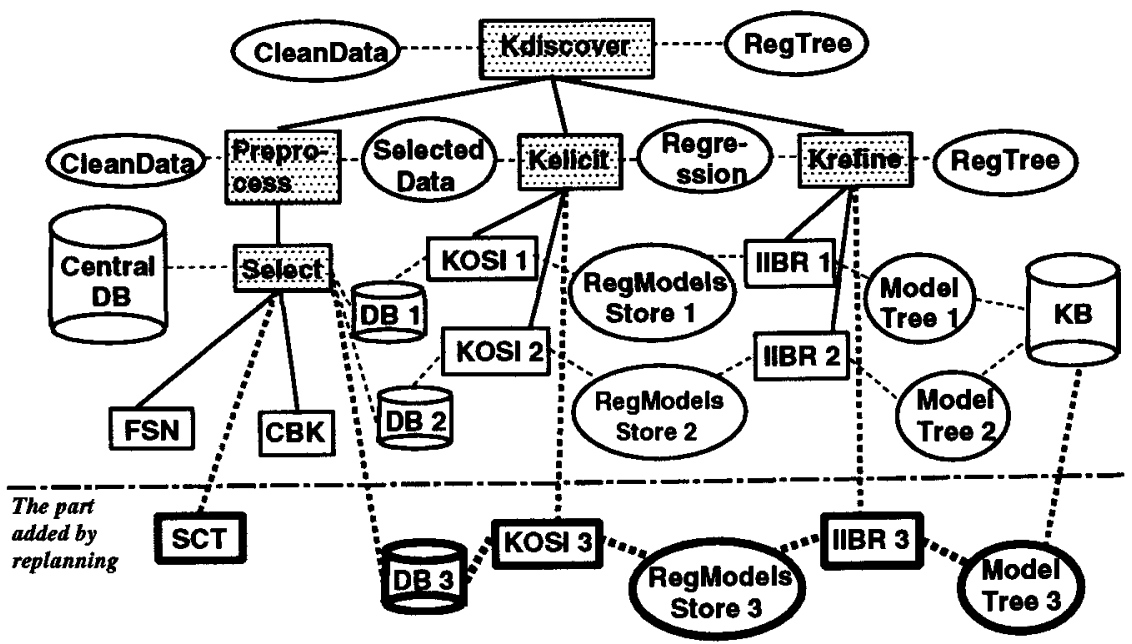

Fig. 2. A sample KDD process plan and replan

learning phase, Select is decomposed into FSN and $C B K$ that can be executed in parallel and cooperatively. The result of the execution of Select (or its subplan) is two subdatabases.

Then the second learning phase Kelicit is under consideration. Here we show how to transforn the In/Out of Kelicit into part of Precond/Effect. The original In/Out specification,

SelectedData $\rightarrow$ Kdiscovered,

is first refined to subtypes:

$\$$ Scope $\rightarrow \$$ Regression,

where $\$$ means unspecified number of. Then, by consulting the current WSD that contains two subdatabases, we have got the precondition "there are two Scopes" and the effect "there will be two regression model stores" (represented as conjunctions of literals). The result of decomposing Kelicit as shown in Figure 2 is that two KOSIs are needed in the subplan of Kelicit to learn from the two subdatabases separately and in parallel. (But in terms of implementation, we may install only one KOSI tool with multiple executions, just as in software development process, one compiler may be used simultaneously to compile several source files). In the third learning phase Krefine, we have a similar situation: there are two IIBRs to refine and manage the knowledge (regression models) discovered by the two KOSIs in parallel. 


\section{Change Management by Incremental Replanning}

The KDD process is a long-term and evolving process. During its lifetime, many kinds of change may occur, hence change management is recognized as an important research issue with practical significance in the field of KDD process.

\subsection{Types of Changes and Management Issues}

We can identify the following kinds of changes (but we do not claim that the list is complete):

- Locil Data Changes in Databases: When the KDD process is planned and executed first time, the original database is used and the discovered regression models are stored for each subdatabases. Later, whenever local data change (a new data item is added, an old data item is deleted/modified, etc.) occurs, the planning and execution process will iterate to find and add new version of regression models to the stores, and each $I I B R$ agent will manage and refine the corresponding tree of regression models. This is a universal and important problem in all real world KDD applications, as the contents of most databases are ever changing.

- Large-Scale and/or Structural Changes in Databases: Some changes in the data could be big and structural, resulting in different decomposition of the central database, for example. In this case, the process plan itself should be changed accordingly. This is called process evolution.

- Changes in the Process Schema: The formal description of all available KDD techniques (i.e. the Agent types) in the KDD system is called the process sch ma. Process schema could change during the lifetime of the KDD process. For example, new KDD techniques can be introduced into the KDD system; existing KDD techniques could become obsolete, or remain in the system but with new parameter settings; new/modified strategies coordinating various discovery steps are adopted; and etc. These changes should be reflected in the process schema accordingly: some new Agent types are added, while some old Agent types are either removed, or modified in their "type-level" attributes (In/Out, Precond/Effect, Decomp). Finally, process schema changes in turn cause process plan changes. That is, we see process evolution here again.

For some of the changes mentioned above, the KDD meta agents (the KDD planner and the KDD controller) presented in the previous section can be further extended to deal with them. For example, if we add the following Monitoring Rule:

Monitoring Rule:

IF there is local change in the databases

THEN restart the process according to the same process plan

With this new (meta-)rule, the databases are under monitoring. Whenever their contents change locally, (a new data item is added, or an old data item is 
deleted/modified), the KDD process is restarted according to the same process plan.

However, in the case of process evolution, changes are difficult to be handled in this way. Because here the problem we are facing is not the re-execution of (part of) the existing plan. Rather, we should replan the KDD process to reflect the changing environment. More precisely, we have the following observations:

- If we insist in solving the problem of process evolution and process replanning by further extending the set of (meta-)rules, our production system will become too complicated. As (Jonsson \& Backstrom 1996) points out, the integration of planning and execution is suitable only for some restricted classes of planning problems (the $3 \mathrm{~S}$ class, for example). As we are not sure if the KDD planning problem in its full-scale can be solved properly by the ever-expanding set of (meta-)rules, we may try to realize replanning as an additional component of the searching strategy of the production system.

- As replanning from scratch is in most cases unpleasant and unnecessary, we need a method to reuse the existing KDD process plan, with local adjustment adapted to the changes. In other word, we need an incremental replanning algorithm.

- The big variety of possible changes does not mean that we need a separate replanning algorithm for each kind of changes. In fact, all possible changes can disturb an existing plan only in the following ways:

- Some new preconditions come in;

- Some old preconditions become unsupported;

- Some old effects become obsolete;

- Decomps of some agents change when new (old) Agent types are added into (renıoved from) the schema;

A general incremental replanning algorithm just needs to consider all these situations and take proper replanning activities.

- Because of the hierarchical planning, the KDD process plan has a hierarchical structure. Incremental replanning always works on a particular part at particular levels of the existing plan, and at a particular time. So we should specify when, where and how to replan.

\subsection{Incremental Replanning for Change Managements}

In light of the above observations, we have designed a general, incremental replanning algorithm. In the following, we present the algorithm in the context of the original coupling mode.

First we recite the searching strategy of the production system implementing the non-linear planning as a non-linear planning algorithm which is called by the KDD controller when it tries to "execute" a high-level KDD agent $A$ : 
ALGORITHM-1: Non-Linear Planning

INPUT: $\quad$ (1) High-level agent $A$ to be decomposed

(2) Current WSD

OUTPUT: $\quad$ Plan of $A$ (a network of agents carrying out A's job)

METHOD:

1. Create AI goal $G$ from the $O u t / E f f e c t$ attributes of $A$, consulting WSD;

2. Build the initial partial-plan of A: $<S T A R T\{W S D\}, G F I N I S H\{\}>$

3. Inspect the current partial-plan $P$ to find all flaws, order them according to heuristics about flaw priority, and store them in $A G E N D A$

4. IF $A G E N D A$ is empty $/ * P$ is flaw-free, hence a plan of $A * /$

THEN stop $/^{*} P$ is returned to the KDD controller */

5. Try to fix up the flaw $A G E N D A[t o p]$ :

IF there is no more applicable (meta-)rule for the flaw

THEN backtrack

ELSE (1) choose (using heuristics) a rule to fix up the flaw;

(2) transform $P$ into a new partial-plan $P^{\prime}$;

(3) set a backtrack point;

6. Inspect the new current partial-plan $P^{\prime}$ to re-adjust $A G E N D A$;

$I^{*}$ remove the fixed flaw and add newly introduced flaws $* /$

7. Goto step4.

Next, we present the incremental replanning algorithm which is also called by the KDD controller. Recall that one of the main tasks of the KDD controller is to monitor the execution of the process plan. Concerning change management, we charge it with the following extra responsibilities:

- Detecting changes in the databases

- Receiving and approving changes in the process schema

- Determining the starting point of replanning - the high-level KDD agent $A$ that is the root of the affected part in the existing, hierarchical plan.

- Calling the replanning algorithm (ALGORITHM-2 below) with agent $A$ and the changes as the input parameters.

ALGORITHM-2: Incremental Replanning

INPUT: (1) High-level agent $\mathrm{A}$ and its existing plan

(2) Changes demanding replanning from $A$

(3) Current WSD

OUTPUT: Re-adjusted plan of $A$, coping with the changes

METHOD:

1. IF there is any change in WSD (databases), or in Out/Effect of $A$ THEN re-create the AI goal G' for $A$;

2. IF there is a change in Decomp of $A$

THEN delete those agents whose types disappear in the new Decomp of $A$;

$/^{*}$ New agents of new types may be added in step 6 below */

3. For each agent $A_{i}$ in the existing plan of $A$ :

IF there is any change in WSD, or in Out/Effect of $A_{i}$

THEN re-adjust Effect of $A_{i}$ according to the change;

$/^{*}$ specially, START will have new WSD as its $E$ ffect */

4. For each agent $A_{i}$ in the existing plan of $A$ :

IF there is any change in WSD, or in In/Precond of $A_{i}$ 
THEN re-adjust Precond of $\mathrm{Ai}$ according to the change;

/* specially, FINISH will have new goal G' as its Precond */

5. Delete all "dead" agents in the existing plan;

$/ *$ an agent supporting no Precond of other agents becomes "dead" */

6. $/^{*}$ Now the existing plan is disturbed, because step 1-4 above have introduced various flaws into it. */

Invoke the planner to resume its work at step 3 of ALGORITHM-1

to find and fix up new flaws, returning a new plan of $A$;

7. For each high-level subagent $H A_{j}$ in the new plan of $A$ :

IF $H A_{j}$ is newly introduced, or though $H A_{j}$ was in the old plan but had not been expanded THEN do nothing here

$/{ }^{*}$ planning of HAj will be done later when controller tries to execute it. */ ELSE Apply this ALGORITHM-2 recursively to $H A_{j}$

$/^{*}$ because $H A_{j}$ may need replanning as well as its parent $A * /$

Note that the replanning algorithm (ALGORITHM-2) is a recursive procedure, and it in turn calls the non-linear planning algorithm (ALGORITHM-1).

Let us look at an example of replanning. Back to Figure 2, supposing that we have got the following events:

- Time-serious data, come implying possible structural changes in the central $D B$

- A new KDD technique $S C T$ (stepwise Chow test to discover structural changes in time-series data) is introduced into the KDD system;

- Decomp of Select type is modified from $<F S N, C B K>$ to $<F S N, C B K, S C T>$

When the KDD controller detects and approves these changes, it determines that the high-level agent Kdiscover in Figure 2 is the starting point of replanning, and calls ALGORITHM-2 to recursively re-adjust the existing, hierarchical plan, resulting in the following changes in the plan, which are marked in Figure 2 by bold lines and boxes:

- The subplan of the Select agent has an additional SCT subagent to discover possible structural changes in time-series data;

- The Select agent has more subdatabases as its output;

- There are more KOSIs in the subplan of Kelicit to learn Regression Models from the new subDBs;

- There are more IIBRs in the subplan of Krefine to build Model Trees from the new Regression Models.

\section{Concluding Remarks}

KDD process and its planning is a new research field, there are more challenging and deeper issues demanding further research. The major contributions of this paper is addressing and solving one of two important issues that have been essentially untouched in KDD literature: 
- Incremental replanning to manage process changes.

- Integrating KDD process planning with process controlling to automate process iteration;

The second problem (process iteration) can be solved by a mechanism integrating process planning with process controlling. We would like to discuss this problem in our other papers. With the issues being properly handled, the GLS system is more complete in KDD process modeling, and more flexible and robust for practical use.

In comparison with related systems, GLS is mostly similar to INLEN [6] where there are also several existing methods of machine learning integrated and a toolkit like in GLS. However, GLS can dynamically plan and organize the KDD process performed in a distributed cooperative mode. As for KDD process planning, so far we have seen in the KDD literature only one paper [8] devoted to this fopic, emphasizing user-guided task-decomposition (corresponding to the hierarchical planning in our context). In contrast, we apply various AI planning techniques to the area of KDD, taking broader view, and addressing deeper issues such as process iteration and change management.

\section{References}

1. Brachman, R.J. and Anand, T. 1996. "The Process of Knowledge Discovery in Databases: A Human-Centered Approach", in Advances in Knowledge Discovery and Data Mining, MIT Press, 37-58.

2. Fayyad, U.M., Piatetsky-Shapiro, G, and Smyth, P. 1996. "From Data Mining to Knowledge Discovery: an Overview", in Advances in Knowledge Discovery and Data Mining, 1-36.

3. Jonsscin, P. and Backstrom, C. 1996. "Incremental Planning", in New Directions in AI Planning, IOS Press, 79-90.

4. Liu, C 1991. "Software Process Planning and Execution: Coupling vs. Integration", Proc. the 3rd Interrational Conference on Advanced Information Systems (CAiSE91), LNCS 498, Springer, 356-374.

5. Liu, C. and Conradi, R. 1993. "Automatic Replanning of Task Networks for Process Evolution in EPOS", Proc. the 4th European Software Engineering Conference (ESEC'93), LNCS 717, Springer, 437-450.

6. Michalski, R.S. et al. 1992. Mining for Knowledge in Databases: The INLEN Architecture, Initial Implementation and First Results. J. of Intell. Infor. Sys., KAP, 1(1):85-113.

7. Russell, S.J. and Norvig, P. 1995. Artificial Intelligence - A Modern Approach Prentice Hall, Inc.

8. Engels, R. 1996. "Planning Tasks for Knowledge Discovery in Databases - Performing TaskOriented User-Guidance", Proc. Second Inter. Conf. on Knowledge Discovery and Data Mining (KDE-96), AAAI Press, 170-175.

9. Zhong, N. and Ohsuga, S. 1995. "Toward A Multi-Strategy and Cooperative Discovery System", Proc. First Inter. Conf. on Knowledge Discovery and Data Mining (KDD-95), 337-342.

10. Zhon;, N., Kakemoto, Y., and Ohsuga, S. 1997a. "An Organized Society of Autonomous Knowledge Discovery Agents", Peter Kandzia and Matthias Klusch (eds.) Cooperative Information Agent.s. LNAI 1202, Springer, 183-194.

11. Zhong, N., Liu, C., Kakemoto, Y., and Ohsuga, S. 1997b. "KDD Process Planning", Proc. Third Inter. Conference on Knowledge Discovery and Data Mining (KDD-97), AAAI Press, 291-294.

12. Zhong, N., Liu, C. and Ohsuga, S. 1997c. "A Way of Increasing both Autonomy and Versatility of a KDD System", Z.W. Ras and A. Skowron (eds.) Foundations of Intelligent Systems, LNAI 1325, Springer 94-105.

13. Zytkow, J.M. 1993. "Introduction: Cognitive Autonomy in Machine Discovery", Machine Learning, K AP, 12(1-3) 7-16. 